\title{
Il contributo di Franco Simone allo studio dell'Umanesimo europeo
}

Lionello Sozzi

\section{(2) OpenEdition}

1 Journals

Edizione digitale

URL: http://journals.openedition.org/studifrancesi/9394

DOI: 10.4000/studifrancesi.9394

ISSN: 2421-5856

Editore

Rosenberg \& Sellier

\section{Edizione cartacea}

Data di pubblicazione: 1 décembre 2007

Paginazione: 499-503

ISSN: 0039-2944

\section{Notizia bibliografica digitale}

Lionello Sozzi, «ll contributo di Franco Simone allo studio dell'Umanesimo europeo», Studi Francesi [Online], 153 (LI | III) | 2007, online dal 30 novembre 2015, consultato il 13 janvier 2021. URL: http:// journals.openedition.org/studifrancesi/9394 ; DOI: https://doi.org/10.4000/studifrancesi.9394

\section{(c) (†) $\odot$}

Studi Francesi è distribuita con Licenza Creative Commons Attribuzione - Non commerciale - Non opere derivate 4.0 Internazionale. 


\section{L'UMANESIMO IN EUROPA}

Presentazione

\section{Il contributo di Franco Simone allo studio dell'Umanesimo europeo}

Una recente antologia di testi umanistici dà finalmente dell'Umanesimo una configurazione europea: non procede per aree circoscritte ma per linee di sviluppo lungo le quali s'incontrano, di volta in volta, Petrarca e il Cusano, Poliziano e Gaguin, Vives e Ficino, John Colet e Budé, Erasmo e Tommaso Moro ${ }^{1}$. A tale visione sovranazionale e unitaria Franco Simone ha dato, coi suoi studi, un contributo fondamentale: se nel corso del Convegno che l'Accademia delle Scienze di Torino gli ha dedicato nel novembre 2006 si sono uditi e ora si leggono in questo numero di «Studi Francesi» interventi che riguardano l'Italia e la Francia, l'area anglo-sassone e quella germanica, la Spagna, i Paesi Bassi e l'Europa orientale, ciò non è solo per rendere omaggio al grande studioso scomparso trent'anni fa, ma anche perché, oggettivamente, i suoi lavori sono il frutto di una radicata convinzione, dell'idea cioè che la civiltà dell'Umanesimo sia nata, più o meno nell'arco degli stessi decenni, in sedi lontane e diverse, come risposta, magari differenziata, a problemi culturali comuni.

Il contributo di Franco Simone allo studio dell'Umanesimo si è svolto in tre fasi, testimoniate da tre opere che oggi, non meno di allora, si segnalano ai nostri occhi insieme per la loro coerenza e la loro particolare fisionomia: La coscienza della Rinascita negli Umanisti francesi, del 1949, Il Rinascimento francese, studi e ricerche, del 1961, e Umanesimo, Rinascimento, Barocco in Francia, del 1968.

Il primo libro incontrò subito il pieno consenso degli studiosi: chi scrive ricorda che nel corso di un seminario tenuto nei primi anni Cinquanta alla Normale di Pisa da Paul Oskar Kristeller, l'illustre erudito già citava La coscienza della Rinascita tra i contributi più importanti usciti in Europa nel giro di quegli anni. Esso getta le basi di una ricerca che riguarda la consapevolezza con cui, sin dalla fine del Trecento o dall'inizio del Quattrocento, gli umanisti francesi guardano al loro tempo come a un'epoca di profondo rinnovamento culturale. Lo studioso affrontava, dunque, un problema di genesi: «Nell'impostazione dei problemi storici e critici - così dirà più tardi - il mio temperamento mi porta a interessarmi alla loro genesi e al loro primo sviluppo». Per ogni modulo critico e schema di giudizio il sicuro intuito e il lungo allenamento gli consentiranno sempre di indicare - così gli scriverà un suo corrispondente tedesco - l'epoca storica e quasi lo strato geologico di appartenenza, come accade al paleontologo, abile nel pervenire a una rigorosa datazione dei suoi reperti. Così, l'idea di un Medio Evo barbarico, di una radicale frattura tra il passato e il presente e di un profondo, determinante rinnovamento, trova allora, a cavallo tra quei due secoli, le 
sue prime e più lontane testimonianze, alla luce dell'insegnamento del Petrarca, del Boccaccio, del Salutati, poi del Poliziano e del Valla, in umanisti come Robert Gaguin e Guillaume Fichet, poi come Jean de Montreuil e Nicolas de Clamanges, ai quali Simone e, successivamente, i suoi allievi danno finalmente il rilievo che meritano, o come Jean Trithème che opera la congiunzione tra umanisti francesi e tedeschi. Qualcosa distingue per altro il senso storico di Italiani e Francesi, non solo per il dissidio che oppone Petrarca a certi letterati d'oltralpe (ad esempio Jean Hesdin), ma anche perché al lungo arco della barbarie medievale di cui parlano i primi si oppone la coscienza dei Francesi di aver già conosciuto un primo periodo di rinascita nel XII secolo. Di un raffinato senso anche linguistico Simone dà prova inoltre, in quel libro, quando ripercorre a ritroso la storia della metafora luce/tenebre risalendo alla sua prima sorgente che è classica (Lucrezio) e, soprattutto, biblica (salmo 111, vangelo di Giovanni, epistole di S. Paolo), oltre che patristica (Ambrogio, Agostino, Girolamo). Ma il ritorno espressivo non nasconde allo studioso l'emergere di profonde diversità: rispetto agli umanisti italiani, ad esempio, molto attenti ai valori del linguaggio, gli umanisti francesi (si pensi a Lefèvre d'Etaples o a Budé, ma anche a Champier o a Lemaire de Belges) insistono sul necessario connubio tra eloquenza e sapienza, cioè tra la riscoperta dei valori filologici, formali, stilistici anche alla luce dei modelli antichi largamente tradotti e la fedeltà ai valori spirituali e religiosi che forse gli Italiani, a loro giudizio, tendono a trascurare: Erasmo definisce Valla «magis rethoricus quam theologus». Fa eccezione, forse, solo Étienne Dolet che porta l'Umanesimo di Budé alle estreme conseguenze poiché vede nella scienza filologica non uno strumento per accedere alla verità religiosa ma un valore in sé, autonomo e autosufficiente, se non addirittura destinato a scuotere le tradizionali certezze alla luce di una premessa che è già razionalistica e antidogmatica, la stessa che, purtroppo, condurrà Dolet a perire sul rogo. Della prefazione ai Commentarii linguae latinae Franco Simone valorizza anche la visione storica europea: come pochi dei suoi contemporanei Dolet fa scoprire la vicinanza tra l'Italia di Bembo, Sadoleto, Pontano e Alciato e la Germania di Reuchlin, Agricola, Melantone, i Paesi Bassi di Erasmo, l'Inghilterra di Moro e di Tunstall, la Spagna di Vives, la Francia di Budé e di Lefèvre, di Champier e di Rabelais. Dolet già vedeva l'Umanesimo europeo in quella luce unitaria e concorde, pur nelle specifiche diversità, in cui anche noi più che mai crediamo come testimoniano il convegno che ci ha riuniti l'anno scorso e ora la presente raccolta di saggi.

Il secondo libro, Il Rinascimento francese, allarga la ricerca a un ambito più vasto, insiste sull'importanza determinante della fortuna francese del Petrarca umanista ma chiarisce anche l'originalità delle prospettive secondo cui lavorano, in Europa, fra gli altri, Erasmo e Budé. Il libro costituisce, oltre al resto, la prima interpretazione originale e organica che la storiografia italiana abbia avanzato per un gruppo di secoli fondamentali nello svolgimento della cultura e delle lettere francesi. Suo cardine è l'idea che l'influenza italiana sul Rinascimento francese non possa venir messa in dubbio, ma che essa sia assai meno vistosa, a un tempo più complessa e più segreta, di quella proposta da un ben noto schema storiografico. La cultura italiana e la nuova filologia penetrarono in Francia non alla fine del Quattrocento (in conseguenza delle spedizioni nella Penisola) ma tramite l'intermediario del Petrarca (il Petrarca del Canzoniere e dei Trionfi, naturalmente, ma soprattutto il Petrarca delle opere latine, dal De remediis alla traduzione in latino della novella di Griselda) e tramite quel cenacolo di Avignone che fu il fulcro di una convergenza altamente significativa per gli sviluppi di tutta la cultura europea: testimonianza emblematica del produttivo impegno con cui quel cenacolo operò è, oltre al resto, lo specifico carattere che assunse, in quegli anni, la costituzione della biblioteca pontificia avignonese; ma significativo è anche, scrive lo studioso, che «tutti i principali rappresentanti della cultura parigina del principio del Quattrocento abbiano avuto modo, nel decennio precedente, di stringere 
rapporti, durante un loro più o meno lungo soggiorno ad Avignone, con l'ambiente francese maggiormente aperto alle idee umanistiche italiane». Tuttavia, l'Umanesimo italiano e petrarchesco non soltanto sfruttò il vasto materiale scrittorio che in Francia costituiva la preziosa eredità dei secoli medievali, ma s'incontrò con una cultura che già per suo conto si veniva evolvendo e che veniva maturando in termini a volte vivacissimi particolari esigenze e originali problemi:

Ai limiti del Medio Evo e del Rinascimento - precisa Simone - non avvenne in Francia l'innesto di spiriti nuovi e di forme originali su di un albero ormai spogliato dei suoi propri frutti. Tra l'antico e il moderno non esistette alcuna rottura; e neppure un deserto, privo di uomini e di opere, in cui operò soltanto un'influenza straniera. Ma anche in Francia, secondo quanto avvenne in quasi tutti i Paesi europei, le idee dell'Umanesimo e del Rinascimento italiano agirono secondo precise e particolari esigenze e non furono né sole, né esclusive e neppure assolute... Tanto per azione diretta quanto per reazione mediata, i Francesi scelsero in Italia gli autori e le opere che meglio servivano a riproporre e risolvere i problemi imposti, di anno in anno, da una cultura in evoluzione lenta ma progressiva, più di quella italiana legata a una tradizione imperiosa, ma non per questo chiusa a un suo avvenire particolare e singolare. L'Umanesimo e il Rinascimento non si realizzarono in Francia nella passiva assimilazione della civiltà italiana. I fiori freschi non sbocciano tra foglie secche. Oltre ogni astrattezza, quei decenni gloriosi rappresentano la storia delle successive soluzioni che soltanto talenti indigeni seppero trovare per i problemi imposti dalla civiltà letteraria francese nel suo graduale sviluppo. Alla soluzione, ma soltanto a questa, cooperarono validamente e sempre in modo determinante, talenti e opere con assoluta libertà scelti nella cultura italiana e in quella fiamminga.

Al panorama vasto e complesso del primo Umanesimo francese Simone dedica le sue pagine più illuminanti, dimostrando come alle più recenti esigenze culturali siano sensibili anche autori considerati di solito come esponenti di una rigida tradizione, come ad esempio Gerson. Rimane aperto, naturalmente, il problema dell'individuazione dei veri oppositori, che pure indubbiamente ci furono:

Quello che per l'ambiente parigino ancora ignoriamo - scrive ancora Simone - è il nome degli oppositori. Se al Salutati poteva corrispondere in Francia Jean de Montreuil o qualcuno dei suoi amici, chi corrispondeva al Dominici? Un'erudizione incerta ci aveva proposto fino a ieri Gerson e Pierre d'Ailly. Dopo che questi dotti sono stati accolti nel campo degli umanisti, l'opposizione è senza rappresentanti. Abbiamo, qui, un campo tutto aperto alle ricerche. Ma sarà bene avvertire che non si potrà conoscere il valore e anche i limiti del primo Umanesimo francese fino a quando queste ricerche non avranno raggiunto dei risultati sicuri.

Contro chi, insomma, il miles Christi di Erasmo deve combattere? Chi sono i falsi fratres, diffusori di una malam doctrinam contro i quali si scaglia Raulin? Sono i seguaci di una disciplina esteriore e formale? O sono i patiti di un ciceronianismo spinto all'estremo? Simone, in ogni caso, apre nuove prospettive di studio e dà un contributo fondamentale alla ricostruzione della battaglia umanistica e alla scoperta della specificità di una cultura insieme innovativa e legata alla tradizione, sensibile, ancora una volta, ai nuovi fatti retorici come al retaggio etico-religioso, propensa a una possibile conciliazione tra teologia e retorica, orientata in direzione di un rigoroso moralismo cristiano e tuttavia ben decisa nel rifiuto di una cultura ancora legata alle Summae e ai Florilegia. Un contributo, occorre dire, cui Simone può giungere grazie anche al suo debito nei confronti di studiosi come Étienne Gilson, Augustin Renaudet e Paul Hazard, il primo per aver reso acuto in lui il senso di una tradizione che non può morire, il secondo perché lo ha abituato all'idea della saldatura tra Umanesimo e problematica religiosa, il terzo per averlo reso sensibile all'idea di mutamento e di crisi. Ma soprattutto, alle sue conclusioni Simone giunge grazie alla sua vastissima 
conoscenza della storiografia sul Rinascimento e della genesi degli schemi storiografici, la stessa che più tardi gli detterà il saggio Per una storia della storiografia letteraria francese, uscito nel 1966 tra le Memorie dell'Accademia delle Scienze di Torino. Ė in quel saggio la convinzione di aver fissato la prima genesi, in Francia, di quello che amava chiamare il «senso della storia», il primo, graduale, faticoso consolidarsi, cioè, di contro alla visione ciclica dei fatti umani, di una visione cumulativa e progressiva, assecondata dal mito della translatio, utile ai Francesi per puntellare ideologicamente il loro affermato predominio culturale europeo.

Il terzo libro, infine, è una raccolta di saggi in qualche modo collaterali che della circolazione delle idee umanistiche dà testimonianze molteplici, anche circoscritte ad aree geografiche ben determinate: l'area fiorentina, con allusione ai rapporti intercorsi tra Salutati, Bruni e Bracciolini e Fichet, Gaguin e Lefèvre d'Etaples, nonché alla presenza a Firenze di autori di primo piano come Commynes, Budé, Rabelais; l'area veneta, con particolare riguardo alla presenza a Parigi di Marsilio da Padova, alla fortuna di Livio e Seneca, alla funzione culturale europea dell'università padovana; l'area lionese, studiata soprattutto per la prodigiosa attività editoriale che vi si svolse nel primo Cinquecento (spicca il nome del tipografo piemontese Baldassarre da Gabiano) e per la fortuna francese che quegli editori assicurarono alle opere di Dante, Petrarca e Boccaccio. Insomma, al centro delle preoccupazioni storiografiche di Simone è la volontà di indagare sul terreno degli scambi, dei confronti, degli influssi, della circolazione dei libri e delle idee ben al di là di quei confini nazionali su cui la storiografia romantica si compiaceva di insistere; sul terreno, anche, della lenta formazione di quegli schemi storiografici, non di rado artefatti e infondati, che per secoli hanno condizionato l'interpretazione delle varie età, dal Medio Evo all'Umanesimo e al Rinascimento, racchiudendole entro categorie di cui Simone segue da vicino la lenta formazione e la perdurante astrattezza. Come si spazientiva quando, durante un esame, interrogato sull'origine e la provenienza di determinate idee, lo studente sprovveduto rispondeva che «erano nell'aria»! «Ma nell'aria non c'è un bel nulla!» esclamava infastidito Simone: per lui contavano le notizie precise sugli incontri e i contatti, sui libri e sulla loro circolazione, sui manoscritti o i documenti di vario genere che potevano confermare o far cadere determinate ipotesi.

Negli ultimi anni della sua attività Simone andava guardando con preminente interesse a una sorta di «mitologia letteraria francese», a una «mitografia gallica» che gli consentiva di pensare alla letteratura non tanto nella sua dimensione espressiva, o creativa e fantastica, quanto come al luogo ideale in cui miti, simboli e schemi mentali si perpetuano e gradualmente si trasmutano. Alcuni degli anelli salienti di quella mitologia - la visione mitizzata delle guerre d'Italia, l'immagine mitica delle tre corone, o quella di Venezia come terra della libertà, o il reditus regni Francorum ad stirpem gallicam, o un'altra celebre immagine mitica, quella dei nani sulle spalle dei giganti, esemplare per l'idea, che in sé racchiude, dell'avanzamento storico - costituivano i motivi ricorrenti non solo degli scritti, ma anche delle animate conversazioni del Maestro scomparso. Altre guide, altri modelli, in quest'ultima fase della sua attività, s'imponevano alla sua attenzione: Barthes, che non esitava a invitare a Torino, Lévi-Strauss, le cui idee pensava di poter utilizzare per la sua «storiografia letteraria francese», Kantorowicz, di cui si vantava giustamente di utilizzare tra i primi in Italia le suggestive proposte, invitando tutti noi, col calore che gli era consueto, a leggere il saggio sul tema del Pro patria mori nel pensiero medioevale o quello sulla sovranità dell'artista nella concezione rinascimentale.

Simone aveva le sue convinzioni ma nutriva anche un assoluto rispetto per le convinzioni altrui e per il contributo scientifico di studiosi anche lontani dalla sua formazione. I seminari che organizzava sulla nouvelle critique o sulla nozione di barocco, ma soprattutto la rivista di «Studi Francesi», con la sua ampia e universalmen- 
te apprezzata 'Rassegna bibliografica', nascevano, in fondo, da questa sua esemplare esigenza di una esauriente e aggiornata informazione, ignara di preclusioni e aperta a ogni nuova proposta. Era un uomo che alla prodigiosa erudizione univa una straordinaria, anche se mai ostentata, sensibilità ai fatti umani, intellettuali e interiori, spirituali, etici, religiosi. L'Umanesimo è per lui una riscoperta dell'uomo, ma al di fuori di ogni retorica romantica egli segue, di quella riscoperta, nei testi e nelle persone, il sotterraneo cammino, le interne contraddizioni, l'intrecciarsi dell'ansia del nuovo col retaggio del passato, le testimonianze più sfumate e incerte, il chiaroscuro più problematico e per noi, oggi, più vivo e attuale. Per questo, oggi, a trent'anni dalla sua scomparsa, lo ricordiamo con commozione e gratitudine. 